\title{
On the Risk of Opening Distributed Keys
}

\author{
Mike Burmester \\ Department of Mathematics, $\mathrm{RH}$ - University of London, \\ Egham, Surrey TW20 OEX, U.K. \\ e-rnail uhah205@vax.rhbnc.ac.uk
}

\begin{abstract}
We describe an insider known-key attack on kcy distribution systems which are based on public keys. This is of a general type and applies to the key distribution system presented by Yacobi at Crypto '90, the Goss system, the Günther system presented at Eurocrypt ' 89 and the key exchange version of COMSET, based on a system presented by Brandt et al. at Crypto ' 89 . The attack is primarily theoretical, in the sense that it assumes that some session keys are leaked or lost. Well designed systems will prevent this. However it could have practical consequences with certain applications (e.g. negotiation of contracts or poor implementations). We discuss the implications and ways to prevent the attack.
\end{abstract}

\section{Introduction}

Matsumoto-Takashima-Imai [21], Yacobi-Shmuely [29] and Yacobi [28] presented a family of variants of the Diffie-Hellman [13] key distribution system which are based on public keys. These are two-party systems which are non-interactive, have a low communication and computation overhead, and offer a good level of security. In particular, the 'non-paradoxical' key distribution system is provably secure against passive attacks and certain types of known-key attacks [28] (provided the Composite Diffie-Hellman problem is hard). In this paper we show that this system is not secure against general known-key attacks. We use an insider attack which is two-pronged, consisting of a passive attack followed by an active known-key attack. In the passive attack the adversary eavesdrops on a conversation. Then the adversary uses a known-key attack to obtain two chosen keys from subsequent sessions. From these, and from the calls exchanged, the adversary computes the key of the eavesdropped session. The attack is general and applies to many other systems including the Goss system [19], the Günther system [20], and the key exchange version of COMSET [9], when the session key is the bitwise exclusive-or of the partial keys.

Known (chosen) key attacks [28] are analogous to chosen-ciphertext attacks for encryptions. With these the cryptanalyst has access to some session keys. As with encryptions, they are the hardest to foil. Various scenarios for known-key attacks are discussed in [12]. These are,

- Negotiation of contracts: After a contract is signed, there is no need to keep secrecy and the keys used for privacy may be revealed. 
- Verification of treaties: To reduce the possibility of hiding a covert message, the key is revealed immediately after the message is authenticated $[2, \mathrm{p} .33]$.

- Jealous spouse: Alice wants to get the secret session key $K_{b c}$ which her spouse Bob used to encrypt a file sent to Carol. She expects to receive a large file from Bob, but will not adhere to the rules of the key exchange protocol. As a result she will not know the session key $K_{b a}$ which she will exchange with Bob. ${ }^{1}$ So when she gets the encrypted file under the session key $K_{b a}$ from Bob she cannot decrypt it. A few days later she claims to have lost the key $K_{b a}$. Bob has no reason to refuse to give her this key.

The robustness of a protocol against the loss of a session key has been the subject of many recent investigations (e.g. [28, 3, 24]). Yacobi linked it to the "paradox" for signature schemes [28]. Bellare and Rogaway [3] observed that it is necessary for secure authenticated key exchange. They pointed out that even if an adversary gets hold of a session key, this should effect only the session which that key protects. In particular, it should not be any easier for the adversary to compute another session key.

Earlier attacks on key distribution systems such as the mafia attack, or intruder-in-the-middle attack (e.g. [25, 4, 14]), and interleaving attacks [5] (see also [3]), fail to take advantage of the scope of known-key attacks. Typically, they lead to disclosure of secret information which subsequently can be used to obtain session keys, or to impersonate. Our attack is different. The adversary is an insider who succeeds in computing the session key of an earlier conversation, from subsequent sessions with the parties involved in the conversation.

Van Oorschot [27] compared the formal goals of the Goss and Günther systems ${ }^{2}$ by using an extended version of the authentication logic of Burrows, Abadi and Needham $[7,1]$ (see also $[18,8,17,16]$ ) and found that they are essentially the same. One of the main features highlighted was the fact that these systems provide only implicit key authentication, i.e. the session key is not authenticated until both parties prove knowledge of it by using it in a subsequent communication. It may seem that that this is not a serious threat $[27, p .241]$, since even though an adversary can replay old messages and complete fraudulent protocol runs, no real advantage is gained because the resulting key cannot be computed. ${ }^{3}$ But if some keys are leaked or lost then it is a threat. Indeed with our attack the adversary uses knowledge gained from leaked keys of fraudulent runs to compute the session key of an earlier honest run.

The organization of this paper is as follows. In Section 2 we describe our attack and show how it can be applied to the Yacobi system. In Section 3 we extend it to the Goss [19] system, the Günther [20] system, and to the key exchange version of COMSET [9]. In Section 4 we analyze the attack and discuss ways to foil it. We conclude with remarks.

${ }^{1}$ By doing so she hopes to gain some knowledge about $K_{b c}$. We shall see that she may succeed in some cases.

2 Yacobi's scheme was not analyzed, but it is similar.

${ }^{3}$ Diffie-Oorschot-Wiener warn about a threat to the Station-to-Station protocol when the signatures are not encrypted [14, p. 116]. However in this case an intruder can easily hijack the key and we do not have key authentication. 
Remark. It is important to differentiate between theoretical and practical attacks. The attack described in this paper is primarily theoretical since well designed implementations of key distribution systems will prevent session keys being disclosed or lost. Indeed in virtually all real systems the users do not know their encryption keys, and only interact through well defined interfaces. However one should be wary, particularly with poor implementations, or with applications in which the session keys are eventually disclosed (e.g. with negotiations of contracts).

\section{Cryptanalysis of the Yacobi System}

We first consider the 'non-paradoxical' key distribution system presented by Yacobi [28] (see also [21]) and show how our attack applies to it. In the following sections we will show that the attack can be extended to other systems.

\subsection{The Yacobi System}

This system uses a discrete logarithm setting with composite modulus $m=p q$, $p, q$ large secret primes, and base $\alpha \in Z_{m}^{*}$ of large order. Each user $U_{\ell}$ has a secret key $s_{\ell} \in Z_{m}$ and a public key $P_{\ell}=\alpha^{s_{\ell}} \bmod m$. When $U_{i}, U_{j}$ want to establish a common session key they select random exponents $e_{i}, e_{j} \in Z_{m}$, respectively, and exchange calls $R_{i}=\alpha^{e_{i}} \bmod m, R_{j}=\alpha^{e_{j}} \bmod m$. The session key is $K_{i j} \equiv R_{i}^{s_{j}} \cdot P_{i}^{{ }^{e_{j}}} \equiv R_{i}^{{ }^{s}} \cdot R_{j}^{s_{i}} \equiv P_{j}{ }^{e_{i}} \cdot R_{j}^{s_{i}}(\bmod m)$, which both $U_{i}$ and $U_{j}$ can compute. Observe that the session key is the modular product of the 'partial keys' $R_{i}{ }^{3} \bmod m$ and $R_{j}^{{ }^{3}} \bmod m$, which both parties can compute.

The Yacobi system is secure against ciphertext-only attacks by a passive eavesdropper if the Composite Diffie-Hellman problem is hard [28]. Under the same cryptographic assumption it is also secure against certain types of knownkey attacks [28]. However these are rather limited in scope [12].

\subsection{Cryptanalysis of the Yacobi System}

Let $A, B$ and $C$ be users whose secret and public keys are $\left(s_{a}, P_{a}\right),\left(s_{b}, P_{b}\right)$, and $\left(s_{c}, P_{c}\right)$ respectively. $\tilde{A}$ is a dishonest user who does not follow the prescribed protocol, but knows the secret key $s_{a}$ of $A . B$ and $C$ execute the prescribed protocol.

In the following attack $\widetilde{A}$ eavesdrops on a conversation of $B$ and $C$. Subsequently, from separate conversations with $B$ and $C$, and by using a known-key attack, she will compute the session key of $B$ and $C$. 


\subsection{The Triangle Attack}

Phase 1. $\tilde{A}$ eavesdrops on a conversation of $B$ and $C$. Let $R_{b}, R_{c}$ be the calls exchanged and let $K_{b c} \equiv R_{b}^{s_{c}} \cdot R_{c}^{s_{b}}(\bmod m)$ be the session key. $\widetilde{A}$ wants to compute this key. She gets $R_{b}, R_{c}$ by eavesdropping.

Phase 2. $B$ exchanges a key with $\widetilde{A}$. $\widetilde{A}$ uses the call $R_{c}$. Let $R_{b}^{\prime}$ be the call of $B$ and let $K_{b a} \equiv R_{b}^{\prime s_{a}} \cdot R_{c}{ }^{s_{b}}(\bmod m)$ be the session key of $B$.

Phase 3. $C$ exchanges a key with $\tilde{A}$. $\tilde{A}$ uses the call $R_{b}$. Let $R_{c}^{\prime}$ be the call of $C$ and let $K_{c a} \equiv R_{c}^{\prime s_{a}} \cdot R_{b}{ }^{{ }^{c}}(\bmod m)$ be the session key of $C$.

Event 1. Opening the key $K_{b a}$. Suppose that the session key of $B, K_{b a}$, is revealed to $A$.

Event 2. Opening the key $K_{\mathrm{ca} a}$. Suppose that the session key of $C, K_{c a}$, is revealed to $\widetilde{A}$.

Computation. $\widetilde{A}$ computes the key of $B$ and $C$. $\widetilde{A}$ now computes:

- the 'partial key' $R_{c}^{s_{b}} \equiv R_{b}^{\prime-s_{a}} \cdot K_{b a}(\bmod m)$,

- the 'partial key' $R_{b}^{s_{c}} \equiv R_{c}^{\prime-s_{a}} \cdot K_{c a}(\bmod m)$, and finally

- the session key $K_{b c} \equiv R_{b}^{s_{c}} \cdot R_{c}^{s_{b}}(\bmod m)$.

Observe that in Phase 2 and Phase $3, \widetilde{A}$ cannot compute the keys $K_{b a}$ and $K_{c a}$ by herself, unless the Composite Diffie-Hellman problem is feasible (she does not know the discrete logarithms of $R_{c}$ and $R_{b}$ ).

Remark. The conventional definition of a known key attack relates to a situation in which an old session key has leaked or is lost. For example, a session key that was used to encrypt a file is stored in a place that was supposed to be safe, but has leaked. Prior to using the session key, the parties use a handshake protocol to authenticate it. In the triangle attack, $B$ and $C$ initiate Phase 2 and Phase 3 respectively because they want to send files to $\widetilde{A}$. The session keys they 'exchange' are then used to encrypt the files. These keys are leaked to $\widetilde{A}$, who then computes the session key of $B$ and $C$. Observe that the Yacobi 'handshake' is non-interactive and provides only implicit authentication ( $\widetilde{A}$ has sent no file and is not authenticated).

\subsection{Hiding the Attack}

The attack can be prevented by having the parties keep a cache of received old calls $R_{i}$, and requesting fresh calls for each new session. We discuss two methods to hide the triangle attack, based on a variant of Moore's attack for RSA signatures $[10,11,22]$ ).

\section{Modification 1}

Instead of using the old calls $R_{c}$ and $R_{b}$ in Phase 2 and Phase $3, \widetilde{A}$ uses

$$
\hat{R}_{c} \equiv R_{c} \cdot \alpha^{t_{c}}(\bmod m) \text { and } \hat{R}_{b} \equiv R_{b} \cdot \alpha^{t_{b}}(\bmod m),
$$


where $t_{c}, t_{b}$ are randomly selected in $Z_{m}$ by $\widetilde{A}$. Then $K_{b a} \equiv R_{b}^{\prime s_{a}} \cdot \hat{R}_{c}^{s_{b}}(\bmod m)$ and $K_{c a} \equiv R_{c}^{\prime s^{a}} \cdot \hat{R}_{b}^{s^{c}}(\bmod m)$, where $R_{b}^{\prime}$ and $R_{c}^{\prime}$ are the calls of $B$ and $C$. In this case $\widetilde{A}$ computes $\hat{R}_{c}^{s_{b}} \equiv R_{b}^{\prime-s_{a}} \cdot K_{b a}(\bmod m)$ and $\hat{R}_{b}^{s_{c}} \equiv R_{c}^{\prime-s_{a}} \cdot K_{c a}(\bmod m)$. From these, and from (1), she gets the partial keys

$R_{b}^{s_{c}} \equiv \hat{R}_{b}^{s_{c}} \cdot \alpha^{-t_{b} s_{c}} \equiv \hat{R}_{b}^{s_{c}} \cdot P_{c}^{-t_{b}}(\bmod m), R_{c}^{s_{b}} \equiv \hat{R}_{c}^{s_{b}} \cdot \alpha^{-t_{c} s_{b}} \equiv \hat{R}_{c}^{s_{b}} \cdot P_{b}^{-t_{c}}(\bmod m)$,

from which she computes the session key $K_{b c} \equiv R_{b}^{s_{c}} \cdot R_{c}^{s_{b}}(\bmod m)$ of $B$ and $C$.

\section{Modification 2}

If the modulus is a prime $p$, then instead of using the calls $R_{c}, R_{b}$ in Phase 2 and Phase $3, \tilde{A}$ may use $\bar{R}_{c}=R_{c}^{t_{c}} \bmod p$ and $\bar{R}_{b}=R_{b}^{t_{b}} \bmod p$, where $t_{c}, t_{b}$ are randomly selected in $Z_{p-1}^{*}$. Then $K_{b a} \equiv R_{b}^{\prime s_{a}} \cdot \bar{R}_{c}^{s_{b}}(\bmod p)$ and $K_{c a} \equiv$ $R_{c}^{\prime s_{a}} \cdot \ddot{R}_{b}^{s_{c}}(\bmod p)$, where $R_{b}^{\prime}$ and $R_{c}^{\prime}$ are the calls of $B$ and $C$. So $\tilde{A}$ can compute $\bar{R}_{b}^{s_{c}} \equiv R_{c}^{\prime-s_{a}} \cdot K_{c a}(\bmod p)$ and $\bar{R}_{c}{ }^{s_{b}} \equiv R_{b}^{\prime-s_{a}} \cdot K_{b a}(\bmod p)$, and hence the partial

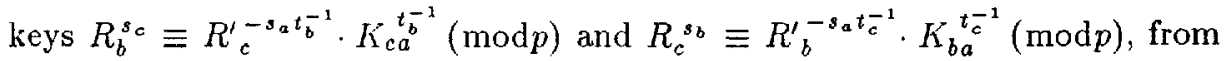
which she gets $K_{b c}$.

\section{Cryptanalysis of Other Key Distribution Systems}

\subsection{The Goss System}

This system [19] is similar to the Yacobi system except that the modulus is a prime $p$, and the session key is the bitwise exclusive-or of the partial keys, instead of their product. That is $K_{i j}=\left(R_{i}^{s^{j}} \bmod p\right) \oplus\left(R_{j}^{s^{i}} \bmod p\right)$.

\section{The Attack}

This is essentially the same as for the Yacobi system. In this case $\widetilde{A}$ computes the session key $K_{b c}$ of $B$ and $C$ from the partial keys $R_{b}^{{ }^{c}} \bmod p=K_{c a} \oplus\left(R_{c}^{s^{s_{a}}} \bmod p\right)$ and $R_{c}{ }^{s_{b}} \bmod p=K_{b a} \oplus\left(R_{b}^{\prime s_{a}} \bmod p\right)$. Both modifications in Section 2.4 apply to this scheme as well.

\subsection{The Günther System}

This system [20] uses ElGarnal signatures [15] for authentication. It is similar to the Yacobi system but with a prime modulus $p$. A trusted center $T$ with secret key $x, 1 \leq x \leq p-1$, and public key $Y=\alpha^{x} \bmod p$, issues each user $U_{\ell}$ with a distinguishing identifier $D_{\ell}$ and an ElGamal signature $\left(P_{\ell}, s_{\ell}\right)$ of $D_{\ell}$. Thus $P_{\ell}=\alpha^{k_{\ell}} \bmod p$, where $k_{\ell} \in Z_{p-1}$ is a random exponent with $\operatorname{gcd}\left(k_{\ell}, p-1\right)=1$, and $s_{\ell}$ is obtained by solving $h\left(D_{\ell}\right) \equiv x P_{\ell}+k_{\ell} s_{\ell} \bmod (p-1)$, where $h(\cdot)$ is a suitable hash function. $P_{\ell}$ is the public key of $U_{\ell}$ and $s_{\ell}$ the secret key. Observe that anyone can compute

$$
P_{\ell}^{s_{\ell}} \equiv \alpha^{k_{\ell \ell}} \equiv \alpha^{h\left(D_{\ell}\right)-x \cdot P_{\ell}} \equiv \alpha^{h^{\left(D_{\ell}\right)}} Y^{-P_{\ell}}(\bmod p)
$$


When $U_{i}$ wants to establish a session key with $U_{j}$ it selects a random exponent $e_{i}$, computes $R_{i}=P_{j}^{e_{i}} \bmod p$, and sends $D_{i}, P_{i}$ and $R_{i}$ to $U_{j}$. Then $U_{j}$ selects a random $e_{j}$, computes $R_{j}=P_{i}{ }^{e_{j}} \bmod p$, and sends $D_{j}, P_{j}$ and $R_{j}$ to $U_{i}$. The session key is $K_{i j} \equiv R_{i}^{s_{j} j} \cdot\left(P_{i}^{s_{i}}\right)^{e_{j}} \equiv R_{i}^{s_{j}} \cdot R_{j}^{s_{i}} \equiv\left(P_{j}^{s_{j}}\right)^{e_{i}} \cdot R_{j}^{s_{j}}(\bmod p)$, which both parties can compute by (2).

\section{The Attack}

As with the Yacobi system, $\widetilde{A}$ eavesdrops on a conversation of $B$ and $C$ with calls $R_{b}, R_{c}$ and secret key $K_{b c} \equiv R_{b}^{s_{c}} \cdot R_{c}^{s_{b}}(\bmod p)$. Subsequently she gets $R_{b}^{\prime}, R_{c}^{\prime}$, and computes the partial keys $R_{b}^{s^{c}} \bmod p$ and $R_{c}{ }^{s_{b}} \bmod p$, and hence the session key $K_{b c}$. Again both modifications apply. Furthermore, it is easy to see that the attack extends to the 'perfect forward secrecy' variant [20, pp. 34-35].

\subsection{COMSET (the Key Exchange Version)}

Each user $U_{\ell}$ of COMSET $[9,6]$ selects secretely two appropriate primes $p_{\ell} \equiv$ $3(\bmod 8)$ and $q_{\ell} \equiv 7(\bmod 8)$ and publishes $n_{\ell}=p_{\ell} q_{\ell}$ as its public key. The encryption function of $U_{\ell}$ is $z \rightarrow z^{2} \bmod n_{\ell}$, and the decryption function is $z \rightarrow$ $z^{d_{\ell}} \bmod n_{\ell}$, where $d_{\ell}=\left(\left(p_{\ell}-1\right)\left(q_{\ell}-1\right)+4\right) / 8$. To establish a secret key with $U_{i}$, $U_{j}$ selects a random $x_{j} \in Z_{n_{i}}$ and computes $m_{j} \equiv x_{j}{ }^{2} \bmod n_{i}$. Then $U_{j}$ sends $U_{i}$ the encryption $r_{j} \equiv m_{j}{ }^{2} \bmod n_{i}$ together with a validator $v_{j}$ of $m_{j}$ which consists of the $\left|n_{i}\right| / 4$ least significant bits of $m_{j} . U_{i}$ decrypts $r_{j}$ to get $m_{j}{ }^{\prime}=r_{j}{ }^{d_{i}} \bmod n_{i}$. It checks the validators of $m_{j}{ }^{\prime}$ and $n_{i}-m_{j}{ }^{\prime}\left(\bmod n_{i}\right)$, and takes $m_{j}{ }^{\prime \prime}$ to be the one whose validator is $v_{j}$. Then it sends $U_{j}$ the next $\left|n_{i}\right| / 4$ significant bits of $m_{j}{ }^{\prime \prime} . U_{j}$ checks these. If they are the same as the corresponding bits of $m_{j}$ then the authentication of $U_{i}$ is successful. The secret (partial) key is $\left[m_{j}\right]=\left[m_{j}{ }^{\prime \prime}\right]$, where $\left[z_{j}\right]$ is an appropriate substring of $z_{j}$. For mutual authentication $U_{j}$ must also be authenticated. The same protocol is used (the processes can be executed in parallel). The common session key is the bitwise exclusive-or $K_{i j}=\left[m_{i}\right] \oplus\left[m_{j}\right]$ of the partial keys.

\section{The Attack}

As with the Yacobi system, $\widetilde{A}$ eavesdrops on a conversation of $B$ and $C$ with calls $r_{b} \equiv m_{b}^{2} \bmod n_{c}$ and $r_{c} \equiv m_{c}^{2} \bmod n_{b}$, and secret key $K_{b c}=\left[m_{b}\right] \oplus\left[m_{c}\right]$ in Phase 1. In Phase 2 she gets the call $r_{b}^{*}=m_{b}^{*} \bmod n_{a}$. Since $n_{a}$ is her own key, she can compute $m_{b}{ }^{*}$ and hence send $B$ the appropriate substring for authentication. In Phase 3 she gets the call $r_{c}{ }^{*}=m_{c}{ }^{* 2} \bmod n_{a}$, computes $m_{c}{ }^{*}$, and sends $C$ the appropriate substring for authentication. Then she gets the keys $K_{c a}=\left[m_{c}^{*}\right] \oplus\left[m_{b}\right]$ and $K_{b a}=\left[m_{b}{ }^{*}\right] \oplus\left[m_{c}\right]$. Finally she computes the partial key $\left[m_{b}\right]$ and the partial key $\left[m_{c}\right]$ from which she gets the session key $K_{b c}$ of $B$ and $C$. For this scheme the modifications in Section 2.4 do not apply.

\section{Repairing the Systems}

When a cryptographic algorithm is used to solve a security problem, it is implemented within the framework of a protocol which ensures that certain levels of 
security required by the system are attained. If this goal is not met, this is either because the cryptographic algorithm is defective or because the protocol procedures are inappropriate. Many of the proposed Diffie-Hellman non-interactive variants have succumbed to attacks by passive and/or active adversaries. However their failure can be attributed to a large extent to the protocol procedures, and can be controlled by specifying a different implementation.

The simplest way to foil the triangle attack is to refuse to willingly reveal session keys to anyone. A sensible precaution is to ensure that session keys are destroyed immediately after the session is ended. This is one of the fundamental design principles for secure key management (and has been widely recognized). Alternatively, the users can be prevented from knowing their own session keys. To implement this, the key distribution algorithm can be encapsulated in a tamper-resistant device [4]. To enforce security the encryption algorithm must also be encapsulated.

Another way to foil the triangle attack is to modify the key distribution systems so as to get key confirmation. This can be done by having each user send an additional message which employs the established session key, e.g. via encryption or a MAC [14]. Then $\widetilde{A}$ cannot complete Phase 2 and Phase 3 of the attack in Section 2.3 , and therefore $B$ and $C$ will destroy the session keys. However this is at the cost of making the key distribution systems interactive: the messages the users send to each other are now dependent (COMSET is already interactive).

Alternatively one can take the session key to be the hash $h\left(K_{i j}\right)$, where $h(\cdot)$ is a suitable hash function, or, the hash of the concatenation of the partial keys. This will foil the triangle attack. However, it must be pointed out that the fact that this attack is foiled does not guarantee that the system is secure (e.g. from other known key attacks). The problem with these modifications is that the adversary gains some knowledge from fraudulent runs when their session keys are leaked, later on (this is not the case with honest runs when a uniform distribution is used, and the 'view' of the adversary can be simulated [12]). It is sensible to prevent such runs e.g. by requiring key confirmation.

Finally one can strengthen the algorithm. This problem is addressed in [12]. The solution proposed there is to require, additionally, that the users $U_{i}, U_{j}$ prove to each other that they know the discrete logarithm of their calls $R_{i}, R_{j}$, by using an interactive zero-knowledge proof. This will prevent fraudulent runs ( $B$ and $C$ will not compute their session key unless they are convinced that $\widetilde{A}$ knows the discrete logarithms of her calls), and furthermore no knowledge about the session keys is leaked to the adversary ( $\tilde{A}$ can simulate her view).

\section{Conclusion}

We have described a general known-key attack for key distribution systems for which the parties compute the session key from partial keys which are established non-interactively, and for which, given the session key and one partial key, the other partial key can be recovered. From our discussion it follows that: 
- Revealing keys to legitimate holders may be harmful.

- Ensuring that session keys are deleted at the end of a session is a sensible precaution.

- Implicit key authentication can be dangerous.

- Taking the session key to be the bitwise exclusive-or, or product, of partial keys may be risky.

- It should not be possible to complete a fraudulent session.

- If files are to be stored, then the plaintext should be encrypted with a different key.

The following remains an open problem:

- Do there exist practical non-interactive proven secure ${ }^{4}$ key distribution systems?

\section{Acknowledgements}

The author would like to thank Kaisa Nyberg for many helpful (and enjoyable) discussions, and in particular for pointing out that the triangle attack extends to COMSET. Many others have contributed in different ways to this investigation, in particular, Ivan Damgård, Whit Diffie, Fred Piper, Peter Landrock, Yacov Yacobi, and Paul van Oorschot. The author is particularly grateful to the referees for several constructive comments on the submitted version.

\section{References}

1. M. Abadi, M. Tuttle: A semantics for a logic of authentication. In: Proc. ACM Symp. on Principles of Distributed Computing. ACM Press 1991, pp. 201-216

2. J.A. Adam: Ways to verify the U.S.-Soviet arms pact. IEEE Spectrum, pp. 30-34 (1988)

3. M. Bellare and P. Rogaway: Entity authentication. To appear in: Crypto '93, Proceedings, Lecture Notes in Computer Science. Berlin: Springer

4. S. Bengio, G. Brassard, Y. G. Desmedt, C. Goutier, J-J. Quisquater: Secure implementations of identification systems. Journal of Cryptology 4(3), 175-183 (1991)

5. R. Bird, I. Gopal, A. Herzberg, P. Jansen, S. Kutten, R. Molva, and M. Yung: Systematic design of two-party authentication protocols. In: J. Feigenbaum (ed.): Advances in Cryptology - Crypto '91, Proceedings. Lecture Notes in Computer Science 576. Berlin: Springer 1992, pp. 44-61

6. J. Brandt, I. Damgård, P. Landrock, and T. Pedersen: Zero-knowledge authentication scheme with secret key exchange. In: S. Goldwasser (ed): Advances in Cryptology - Crypto '88, Proceedings. Lecture Notes in Computer Science 403. Berlin: Springer 1990, pp. 583-588

${ }^{4}$ A system is proven secure if breaking it (e.g. by using a known key attack) is as hard as solving a (believed to be) hard cryptographic 'reference' problem such as, factoring, the discrete logarithm, the Diffie-Hellman problem, etc. For practical systems this seems to be the best achievable level of security. 
7. M. Burrows, M. Abadi, R. M. Needham: A logic of authentication. ACM Trans. Computer Systems 8, 18-36 (1990)

8. P-C. Cheng, V. Gligor: On the formal specification and verification of multiparty session protocols. In: Proc. IEEE Sym. on Research in Security and Privacy. IEEE Press 1990, pp. 216-233

9. COMSET. Ripe Integrity Primitives. Final Report of RACE Integrity Primitives Evaluation (R1040). June 1992, pp. 191-201

10. G.I. Davida: Chosen signature cryptanalysis of the RSA (MIT) public key cryptosystem. Tech. Report TR-CS-82-2, University of Wisconsin-Milwaukee, October 1982.

11. D.E.R. Denning: Digital signatures with RSA and other public-key cryptosystems. Comm. ACM 27(4), 388-392 (1984)

12. Y. Desmedt, M. Burmester: Towards practical 'proven secure' authenticated key distribution. In: Proceedings 1st ACM Conference on Computer and Communication Security, Fairfax, Virginia. ACM Press, November 1993, pp. 228-231

13. W. Diffie, M.E. Hellman: New directions in cryptography. IEEE Trans. Inform. Theory, I'T-22(6), 644-654 (1976)

14. W. Diffie, P.C. van Oorschot, M.J. Wiener: Authentication and authenticated key exchanges. Designs, Codes and Cryptography 2, 107-125 (1992)

15. T. ElGamal: A public key cryptosystem and a signature scheme based on discrete logarithms. IEEE Trans. Inform. Theory 31, 469-472 (1985)

16. K. Gaarder, E. Snekkens: Applying a formal analysis technique to the CCITT X.509 strong two-way authentication protocol. J. Cryptology 3(1), 81-98 (1991)

17. V. Gligor, R. Kailar, S. Stubblebine, L. Gong: Logics for cryptographic protocols - virtues and limitations. In: Proc. IEEE 1991 Computer Security Foundations Workshop. Franconia, New Hampshire 1991

18. L. Gong, R. Needham, R. Yalom: Reasoning about belief in cryptographic protocols. In: Proc. 1990 IEEE Symp. on Security and Privacy, Oakland, California. IEEE Press 1990, pp. 234-248

19. K.C. Goss: Cryptographic method and apparatus for public key exchange with authentication. U.S. Patent 4,956,863, Granted Sept 11, 1990.

20. C.G. Günther: An identity-based key exchange protocol. In: J-J. Quisquater, J. Vandewalle (eds.): Advances in Cryptology, Eurocrypt '89. Lecture Notes in Computer Science 434. Berlin: Springer 1990, pp. 29-37

21. T. Matsumoto, Y. Takashima, H. Imai: On seeking smart public key distribution systems. The Transactions of the IECE of Japan E69(2), 99-106 (1986)

22. J.H. Moore: Protocol failures in cryptosystems. Proc. IEEE 76(5), 594-602 (1988)

23. K. Nyberg, R. Rueppel: A new signature scheme based on the DSA giving message recovery. In: Proceedings 1st ACM Conference on Computer and Communication Security, Fairfax, Virginia. ACM Press 1993, pp. 58 61

24. K. Nyberg, R. Rueppel. Weaknesses in some recent key agreement protocols. Electronics Letters, 30(1), 26-27 (1994)

25. R. L. Rivest, A. Shamir: How to expose an eavesdropper. Comm. ACM 27(4), pp. 393-395 (1984)

26. G.J. Simmons: How to ensure that data aquired to verify treaty compliance are trustworthy. In: G.J. Simmons (ed.): Contemporary Cryptology. New York: IEEE Press 1992, pp. 615-630.

27. P.C. van Oorschot: Extending cryptographic logics of belief to key agreement protocols. In: Proc. 1st ACM Conference on Computer and Communications Security, Fairfax, Virginia. ACM Press 1993, pp. 232-243 
28. Y. Yacobi: A key distribution paradox. In: A.J. Menezes, S.A. Vanstone (eds.): Advances in Cryptology - Crypto '90, Proceedings. Lecture Notes in Computer Science 537. Berlin: Springer 1991, pp. 268-273

29. Y. Yacobi, Z. Shmuely: On key distribution systems. In: G. Brassard (ed.): Advances in Cryptology - Crypto '89, Proceedings. Lecture Notes in Computer Science 435. Berlin: Springer 1990, pp. 344-355 